\title{
Comunicación y negociación como predictores del uso del condón en hombres privados de la libertad ${ }^{1}$
}

\author{
Communication and negotiation as predictors \\ of the use of the condom in incarcerated men
}

\author{
Ricardo Sánchez-Medina, Anabel de la Rosa-Gómez, \\ David Javier Enríquez-Negrete y Dulce María Velasco-Hernández²
}

Citación: Sánchez-Medina, R., de la Rosa G., A., Enríquez N., D.J. y Velasco H., D.M. (2020).

Comunicación y negociación como predictores del uso del condón en hombres privados de la libertad. Psicología y Salud, 30(2), 243-251. https://doi.org/10.25009/pys.v30i2.2658.

\section{RESUMEN}

El objetivo del presente estudio fue identificar si la comunicación sobre temas sexuales y el estilo de negociación de colaboración-equidad predicen el uso del condón en las relaciones sexuales de hombres privados de la libertad, para lo cual se retomó el modelo de Información, Motivación y Habilidades Conductuales como marco de referencia. Se evaluó a 687 hombres internados en un centro varonil de reinserción social de la Ciudad de México. Se encontró que la comunicación y el estilo de negociación para usar condón centrado en la colaboración y equidad son variables que predicen el uso de este preservativo en las relaciones sexuales. Las variables de información y motivación que predicen la comunicación son la intención, la autoeficacia, las actitudes y las creencias hacia el uso del condón, así como los conocimientos sobre el VIH/sida; en cambio, los predictores del estilo de negociación fueron las creencias, la autoeficacia y las actitudes hacia el uso del condón y la información sobre el VIH. Los resultados indican la necesidad de desarrollar las habilidades centradas en la comunicación con la pareja y los modos de negociar el uso del condón en las relaciones sexuales en los programas de intervención en dicha población.

Palabras clave: Hombres; Condón; Prevención; Habilidades; Hombres encarcelados.

\begin{abstract}
The objective of the present study was to identify whether communication on sexual issues and the collaborative-equity negotiation style predict condom use in male prison inmates' sexual relations. The frame of reference was the model: Information-motivation-skills-behavior. A total of 687 prison inmates in Mexico City were evaluated. Results revealed that communication and negotiation styles for condom use focused on collaboration and equity as variables predicting condom use in sexual relationships. Intention, self-efficacy, attitudes, and beliefs towards condom use, as well as knowledge about HIV/AIDS, were variables predicting communication on information and motivation. On the other hand, predictors for negotiation styles were beliefs, self-efficacy and attitudes towards condom use and HIV information. Results indicate the need of incorporating intervention programs for the development of skills focused on communication with sexual partners, and the ways for negotiating the use of condoms in sexual relationships in such population.
\end{abstract}

Keywords: Men; Condom; Prevention; Skills; Incarcerated men.

\footnotetext{
${ }^{1}$ Investigación realizada gracias al Programa UNAM-DGAPA-PAPIIT IA304317. Artículo recibido el 19 de diciembre de 2018 y aceptado el 26 de marzo de 2019.

${ }^{2}$ Facultad de Estudios Superiores Iztacala de la Universidad Nacional Autónoma de México, Av. de los Barrios Núm. 1, Los Reyes Iztacala, 54090 Tlalnepantla, Edo. de México, México, tel. (55)56-23-13-33, ext. 39855, correos electrónicos: ricardo.sanchez@iztacala.unam, anabel.delarosa@iztacala.unam, david.enriquez@iztacala.unam y dulce.velasco@iztacala.unam.
} 


\section{INTRODUCCIÓN}

$\mathrm{L}$ a privación de libertad es concebida como el modo en que se despoja a una persona de sus actividades actuales e incluso de sus derechos, obligándolo a cumplir una sentencia en un centro penitenciario por el delito cometido. A partir de ese momento, el sujeto ya no puede decidir nada respecto a su vida, sino que serán otros quienes lo harán (Escaff, Estévez, Feliú y Torrealba, 2013).

Rubio (2013) señala que, para un ser humano, estar privado de la libertad puede resultar una de las experiencias más traumáticas de su vida, ya que es separado de su familia, pareja, amistades, trabajo o escuela; es decir, del entorno social en el que se encontraba antes de su ingreso a la prisión. Este proceso tiende a influir de manera negativa en las personas física y psíquicamente en su adaptación al nuevo medio (Añaños y Jiménez, 2016).

La población privada de la libertad (PPL en lo sucesivo) carga un estigma social. A pesar de los importantes avances del sistema penitenciario en el país, aún se siguen observando casos de exclusión social, laboral y sanitaria (Añaños y Jiménez, 2016; Cutiño, 2015), pues las prácticas permitidas a esta población vulneran no solo su salud, sino también sus derechos fundamentales (Galindo, 2012). Algunas aportaciones de dichos programas, de acuerdo con Cutiño (2015), revelan que, del total de las personas que ingresan a prisión, $11.7 \%$ eran usuarios de drogas, y de ellos, alrededor de 35\% está infectado por el Virus de Inmunodeficiencia Humana (VIH). La prevalencia del VIH y del síndrome de inmunodeficiencia adquirida (sida) en el ámbito penitenciario es mayor que en la población general en la mayoría de los casos, por lo que reducirla es una parte importante de la respuesta nacional (Comisión Nacional de los Derechos Humanos [CNDH], 2012).

Considerando la relevancia de las infecciones de transmisión sexual (ITS), entre ellas el VIH/ sida, dicha Comisión afirma que son un problema de salud pública que se ve agravado por el estigma y la discriminación cuando las personas con VIH y otras ITS se encuentran en internadas en reclusorios.

La salud sexual es un estado de bienestar físico, mental y social que requiere un enfoque po- sitivo y respetuoso de las relaciones sexuales, de modo que las personas tengan la posibilidad de tener experiencias sexuales placenteras, seguras y libres de toda coacción, discriminación y violencia (Organización Mundial de la Salud [OMS], 2018).

La transmisión del VIH obedece en buena medida a las relaciones sexuales desprotegidas, sobre todo entre hombres, incluso más que el uso de equipo de inyección no esterilizado $(\mathrm{CNDH}$, 2012). De acuerdo con las estadísticas del Centro Nacional para la Prevención y Control del VIH y el Sida (CENSIDA) (2018), hasta junio de 2018 los casos notificados de VIH y sida de 1983 a 2018 ascendían a 198,598, y los casos no fatales según su estado de evolución, ascendían a 155,276 (sida, 79,223 y VIH, 76,053 ), de los cuales $82 \%$ correspondían a hombres. Los casos nuevos diagnosticados preliminarmente en 2018 fueron 6,827 (sida, 2,644, y VIH, 4,183); según esas estadísticas, el número de defunciones en México en 1990 fue de 1,503, que en 2017 ascendió a 4,265.

El VIH/sida es una amenaza sanitaria para la población privada de la libertad y un desafío importante para las autoridades encargadas de las instituciones de reinserción social, salud pública y gobiernos en general. Los casos de transmisión por VIH tienden a ser más elevados en los centros de reclusión que fuera de los mismos en todo el mundo, y suelen agravarse con coinfecciones como la hepatitis B y C y la tuberculosis (CNDH, 2012).

La CNDH (2012) afirma que en los centros penitenciarios existen dos vías principales de infección: la primera es a través de los internos adictos a las drogas que comparten las jeringas, y la segunda a prácticas sexuales en condiciones de riesgo, como no usar condón, incluso desde el momento de la reclusión.

El Programa Conjunto de las Naciones Unidas sobre el VIH/sida (ONUSIDA)/y la OMS (2007) indican que todos los centros penitenciarios cuentan con programas de salud; sin embargo, por un lado, hay un abasto insuficiente de condones y, por el otro, recursos humanos escasos que se encarguen de cubrir las necesidades de información sobre prevención; o bien personal no capacitado en el problema del VIH/sida, que si bien ha sido capacitado, influyen en él creencias erróneas respecto 
al miedo irracional de convivir con personas con VIH/sida. Tal situación se caracteriza, pues, por la falta de conocimientos y por la poca sensibilidad hacia esos internos en la mayoría de centros de reinserción.

El uso del condón forma parte de una de las estrategias más exitosas que se han puesto en práctica para fomentar el sexo protegido y así disminuir la incidencia del VIH/sida y otras ITS. Siempre y cuando se le utilice de manera correcta y consistente, el condón representa uno de los métodos más efectivos para el cuidado de la salud sexual (CENSIDA, 2016).

Asare (2015) ha encontrado que el mejor predictor del uso consistente del condón es la intención; sin embargo, ese modelo no considera variables conductuales tales como la comunicación y la negociación, las cuales son también relevantes ya que tienen un impacto en la prevención de las conductas de riesgo (Fishbein, 2000). Así, uno de los modelos que dan cuenta de ello es el de Información-Motivación-Habilidades conductuales (IMB, por sus siglas en inglés). Este modelo sostiene que el que una persona tenga un comportamiento preventivo - como el uso del condón-depende de las habilidades conductuales y de la información y motivación que posea (Fisher y Fisher, 1992).

Respecto a esas habilidades conductales, generalmente se evalúa la capacidad que tiene un individuo para ejecutar una conducta en términos de su eficacia (Kiene et al., 2013; McGrane, Mittal, Elder y Carey, 2016). Autores como Leddy, Chakravarty, Dladla, de Bruyn y Darbes (2016), así como Sánchez, Robles y Enríquez (2015), resaltan la importancia de evaluar las habilidades a partir de lo que las personas hacen y no de qué tan capaces se sienten para hacerlo; por ejemplo, la comunicación que establecen con la pareja sexual predice el tener relaciones sexuales protegidas mediante el uso constante y correcto del condón.

Se ha encontrado que la comunicación con la pareja sexual es un predictor consistente del uso del condón en una relación sexual; sin embargo, según el modelo IMB, se requieren también el conocimiento y la motivación necesarios. Robles, Frías, Moreno, Rodríguez y Barroso (2011) afirman que, para que esta habilidad se demuestre, es necesario ofrecer argumentos que convenzan a la pareja de usar condón, los que pueden estar basados en la información que se tiene respecto a la prevención y transmisión del VIH/sida; mencionan además que cuando las personas cuentan con dichas habilidades, serán más frecuentes sus relaciones sexuales protegidas. Otra conducta que se ha tomado en cuenta en este componente tiene que ver con la negociación del uso del condón, ya que cuando la persona sabe cómo hacerla, será más probable que la utilicen en sus relaciones sexuales (Camilleri, Kohut y Fisher, 2015).

Acerca de los componentes del modelo asociados con la información y la motivación, Uribe, Aguilar, Zacarías y Aguilar (2015) señalan que el uso del condón en las relaciones sexuales está vinculado al desarrollo de ciertos factores psicosociales, como la valoración positiva y la aceptación del condón. Eggers et al. (2015) reportan como predictores del uso adecuado del condón las actitudes favorables, el conocimiento de cómo usarlo y la norma subjetiva.

Uribe et al. (2017) y Robles et al. (2012) han encontrado que la percepción de autoeficacia en el uso del condón es una variable que se vincula con aspectos cognitivos, tales como la intención de usar condón, una buena actitud a ese respecto y la percepción de obtener beneficios al utilizarlo, así mismo, puede estar asociada a las conductas preventivas en el uso adecuado del condón. Icunacuri (2010) detalla que el estilo de negociación sobre el uso del condón centrado en llegar a un acuerdo con la pareja para utilizarlo en sus relaciones sexuales, fue el que más emplearon los sujetos de su estudio; aunque la mayoría reportó tener la intención de usar condón, tendían más a ajustarse a un acuerdo con su pareja para evitar un conflicto. Es así como la intención, las actitudes y las creencias favorables hacia el uso del condón, además de la comunicación con la pareja sobre la sexualidad, son variables de interés asociadas al uso consistente del condón (Icunacuri, 2010; Pinos et al., 2017; Sánchez et al., 2015).

Según Fishbein (2000), las intervenciones para un cambio de comportamiento bien diseñadas, dirigidas y enfocadas en la teoría pueden ser eficaces para reducir VIH/sida y otras ITS. Por lo tanto, el presente estudio tuvo dos objetivos fundamentales: identificar si la comunicación sobre 
temas sexuales y el estilo de negociación de colaboración-equidad predicen el uso del condón en las relaciones sexuales, e identificar qué variables de información y motivación predicen estas habilidades conductuales, de tal forma que se disponga de indicadores para el diseño de programas de intervención.

\section{MÉTODO}

\section{Tipo de estudio}

De acuerdo con la clasificación de Méndez, Namihira, Moreno y Sosa (2001), el presente estudio fue de corte prospectivo (toda la información se recoge de acuerdo a los criterios establecidos a partir de su planeación), transversal (se miden las variables una sola vez, y las características del grupo en un momento dado), descriptivo (se cuenta con una población, la cual se pretende describir en función de un grupo de variables) y observacional (se describe el fenómeno estudiado sin modificar ninguno de los factores que intervienen en el proceso).

\section{Participantes}

La muestra estuvo conformada por 687 hombres recluidos en un centro de reinserción social de la Ciudad de México, a quienes, mediante un muestreo por conveniencia, se invitó a responder un cuestionario (Kerlinger y Lee, 2002). Se trabajó con aquellos que firmaron un formato de consentimiento informado, excluyéndose a quienes no podían responder los instrumentos por problemas de salud física o mental y a quienes no los completaron. La edad promedio de los participantes fue de 27 años. De ellos, 63\% eran solteros, y el porcentaje restante correspondió a casados o en unión libre; $59 \%$ de ellos tenía estudios de secundaria completa únicamente, $1.9 \%$ estudios universitarios concluidos, y el porcentaje restante únicamente estudios primarios. De los participantes, $95.5 \%$ eran heterosexuales, $1.7 \%$ homosexuales y $2.8 \%$ bisexuales. Los delitos que más predominaron en la muestra seleccionada fueron el robo (84\%) y la violación (3\%).

\section{Instrumentos}

Para dar cuenta de las variables sociodemográficas, se solicitaron a los participantes datos personales tales como edad, estado civil, nivel de estudios, número de hijos (si es que los tenían), orientación sexual, ingresos mensuales antes de su ingreso, datos sobre el delito por el que se encontraban privados de la libertad y años de sentencia. También se les preguntó si habían tenido una vida sexual activa y la frecuencia de uso del condón en un periodo de tres meses, lo que respondieron de acuerdo a un formato Likert, con cinco opciones de respuesta (de "nunca" a "siempre").

Para la evaluación de los componentes del modelo se utilizaron los siguientes instrumentos:

\section{Información}

Se utilizó la Prueba de Conocimientos sobre VIH/ sida-ITS-uso de Condón (cf. Robles y Díaz-Loving, 2011), mediante el cual se evaluaron áreas como sintomatología, transmisión y prevención del VIH, en el que se incluyen tres reactivos con los que se exploran los conocimientos sobre las características de las ITS, así como seis reactivos para evaluar los que se tienen sobre el uso del condón mediante el procedimiento de respuesta de "cierto/falso".

\section{Motivación}

Se aplicó la Escala de Creencias sobre el Uso del Condón (cf. Robles y Díaz-Loving, 2011), que consta de ocho reactivos sobre creencias de seguridad y protección y tres de creencias negativas, los que se valoran en una escala Likert que va de 1 ("totalmente en desacuerdo") al 5 ("totalmente de acuerdo"). La consistencia interna en la muestra, evaluada mediante el coeficiente $\alpha$ de Cronbach, fue de 0.84. También se utilizó la Escala de Actitudes hacia el Uso del Condón (cf. Robles y Díaz-Loving, 2011), que incluye cinco reactivos en formato de un diferencial semántico de 1 a 7 , con un coeficiente $\alpha$ de 0.87 en la muestra evaluada. Adicionalmente, se utilizaron dos reactivos para evaluar qué tan probable es que los participantes utilicen condón (intención) y qué tan capaces se perciben para usarlo (autoeficacia) en su próxima relación sexual; ambas preguntas se miden en una escala Likert con siete opciones de respuesta. 


\section{Habilidades conductuales}

Se utilizó la Escala de Comunicación sobre Temas Sexuales (cf. Robles y Díaz -Loving, 2011), que incluye diez reactivos que evalúan la frecuencia con la que los participantes se comunican con su pareja, lo que se responde en una escala Likert que va de 1 ("nunca") a 5 ("siempre"). Su consistencia interna fue de 0.94 .

Por último, se aplicó el Inventario de Estilos de Negociación sobre el Uso del Condón (cf. Robles y Díaz-Loving, 2011), que utiliza seis reactivos centrados en la colaboración-equidad que hacen referencia a acuerdos con la pareja para usar condón. Dicha escala se evalúa mediante una escala tipo Likert con valores que van de 1 ("totalmente en desacuerdo") a 5 ("totalmente de acuerdo"), y tuvo una consistencia interna en la muestra evaluada de 0.85 .

\section{Procedimiento}

Previa autorización del Consejo Técnico de la Subsecretaría del Sistema Penitenciario, se llevó a cabo la aplicación del instrumento con el apoyo de personal de la institución, para lo cual se pidió una reunión por dormitorio. Una vez reunidos los participantes, se les explicaron los motivos de la investigación y se les solicitó la firma del formato de consentimiento informado, señalándoles que los datos proporcionados de ninguna manera los utilizaría la institución. A quienes otorgaron su consentimiento se les entregó un cuadernillo con los instrumentos, así como un lápiz para su llenado. La duración de la aplicación fue de aproximadamente treinta minutos.

\section{Análisis de datos}

Se realizó un modelo de regresión líneal de pasos sucesivos (stepwise), utlizando para ello el paquete estadístico SPSS, v.20, manteniendo solo las variables significativas $(p<0.05)$. Se reportan los datos de $\beta$, el valor $t$ y el IC $95 \%$, lo que en conjunto permite explicar o predecir el valor de las variables dependientes sobre la independiente; también se reportan los valores de $r$ y $\mathrm{r}^{2}$, que indican la proporción de la varianza total de la variable explicada por la regresión. En un primer momento, se utilizó la frecuencia del uso del condón como variable dependiente, y como variables independientes las centradas en las habilidades (frecuencia de la comunicación sobre temas sexuales y estilo de comunicación de colaboración-equidad). En un segundo momento, se efectuaron dos análisis de regresión lineal de pasos sucesivos, utilizándose cada una de las habilidades como variables independientes, y las variables de información y motivación como variables dependientes.

\section{RESULTADOS}

Se llevó a cabo un análisis de regresión para identificar si las habilidades conductuales predecían la frecuencia del uso del condón. En la Tabla 1 se observa que la comunicación sobre temas sexuales con la pareja y los estilos de negociación para usar el condón centrado en la colaboración-equidad predicen su frecuencia de uso en las relaciones sexuales.

Tabla 1. Habilidades conductuales como predictores de la frecuencia del uso del condón.

\begin{tabular}{|c|c|c|c|}
\hline Variable & $\boldsymbol{\beta}$ & $t$ & IC95\% \\
\hline Comunicación sobre temas sexuales con la pareja & .128 & $2.45^{*}$ & {$[.026,-.231]$} \\
\hline Estilos de negociación-colaboración-equidad & .103 & $2.27 * *$ & {$[.014,-.192]$} \\
\hline R 210 & & & \\
\hline $\mathrm{R}^{2} 044$ & & & \\
\hline $\mathrm{F} 1568 * * *$ & & & \\
\hline
\end{tabular}

Con el propósito de conocer qué variables de información y motivación se asocian con dichas habilidades; en la Tabla 2 se presentan los predictores de la comunicación sobre temas sexuales con la pareja, en donde se observa que la inten- ción para usar el condón, la autoeficacia para ello, los conocimientos sobre VIH/sida, las actitudes y creencias favorables hacia el uso del condón aumentan la comunicación sobre temas sexuales con la pareja. 
Tabla 2. Información y motivación como predictores de la comunicación sobre temas sexuales con la pareja.

\begin{tabular}{|c|c|c|c|}
\hline Variable & $\beta$ & $t$ & IC $95 \%$ \\
\hline Intención para usar condón & .072 & $3.49 *$ & {$[.031,-.112]$} \\
\hline Autoeficacia para usar condón & .073 & $3.55^{* *}$ & {$[.032,-.113]$} \\
\hline Conocimientos sobre VIH/sida & .007 & $2.89 * *$ & {$[.002,-.011]$} \\
\hline Actitudes favorables hacia el uso del condón & .064 & $2.46^{* * *}$ & {$[.013,-.115]$} \\
\hline Creencias favorables hacia el uso del condón & .078 & $2.12 * * *$ & {$[006,-.151]$} \\
\hline $\mathrm{R}=.365$ & & & \\
\hline $\mathrm{R}^{2}=133$ & & & \\
\hline $\mathrm{F}=20.7 *$ & & & \\
\hline
\end{tabular}

Por último, los predictores del estilo de negociación de colaboración-equidad sobre el uso del condón fueron las creencias favorables hacia su uso, la autoeficacia para usarlo, los conocimientos sobre VIH/sida y las actitudes favorables hacia su uso (Tabla 3).

Tabla 3. Información y motivación como predictores del estilo de negociación del uso del condón: colaboración-equidad.

\begin{tabular}{|c|c|c|c|}
\hline Variable & $\beta$ & $t$ & IC95\% \\
\hline Creencias favorables hacia el uso del condón & .280 & $6.60^{*}$ & {$[.197,-.363]$} \\
\hline Autoeficacia para usar condón con la pareja & .065 & $3.11 * *$ & {$[.024,-.106]$} \\
\hline Conocimientos sobre VIH/sida & .009 & $3.35^{* * *}$ & {$[.004,-.014]$} \\
\hline Actitudes favorables hacia el uso del condón & .081 & $2.78 * * * *$ & {$[.024,-.139]$} \\
\hline $\mathrm{R}=.365$ & & & \\
\hline $\mathrm{R}^{2}=.128$ & & & \\
\hline $\mathrm{F}=26.1^{*}$ & & & \\
\hline
\end{tabular}

\section{DISCUSIÓN}

El propósito de la presente investigación fue identificar si la comunicación sobre tópicos sexuales con la pareja y el estilo de negociación de colaboración-equidad predicen el uso del condón en las relaciones sexuales, y la vez identificar las variables de información y motivación del modelo IMB (Fisher y Fisher, 1992) que predicen dichas habilidades, de tal forma que se cuente con indicadores que puedan incluirse en los programas de intervención enfocados a promover la salud sexual en PPL.

Se observó que las dos habilidades evaluadas en su conjunto predicen la frecuencia del uso del condón en las relaciones sexuales. Los valores de $\beta$, al ser positivos, indican que para que alguien utilice de manera frecuente el condón en sus relaciones sexuales es necesario que sea alta la frecuencia con la que habla con su pareja sobre la sexualidad y la negociación del uso del condón para de llegar a un acuerdo sobre su uso.

Lo anterior parece relevante debido a que el desarrollo de estas habilidades puede promover que las personas adquieran un comportamiento sexual preventivo, tal y como se ha demostrados en diversas investigaciones (Camilleri et al., 2015; Kiene et al., 2013; Liu et al., 2014).

De igual forma, dicho modelo señala que para que las personas tengan habilidades conductuales es necesario que posean información y que estén motivados para hacerlo, por lo que adicionalmente se buscó conocer las variables que pueden predecir dichas habilidades. Si bien es cierto que pueden utilizarse modelos más complejos para el análisis (Marsh, Morin, Parker y Kaur, 2014), en la presente investigación se buscó hacer una predicción para cada una de las habilidades que permitiera la toma de decisiones respecto a los elementos a considerar, y asimismo establecer un 
pronóstico de lo que podría suceder al modificar los valores de las variables (Austin y Steyerberg, 2015), que en este caso es desarrollar habilidades que aumenten el uso de condón en las relaciones sexuales en hombres privados de la libertad.

Sobre los predictores de la comunicación con la pareja, se encontró que aquellos que tienen la intención de usar el preservartivo en su próxima relación sexual, que se sientan capaces de usarlo, que tienen información sobre los síntomas y de cómo se previene y se transmite el VIH, así como las actitudes y creencias favorables hacia su uso, hará más probable que hablen con su pareja sobre temas sexuales y de cómo protegerse al tener relaciones sexuales. En el caso particular del estilo de negociación del uso del condón, se encontró que para que tratasen de llegar a un acuerdo con su pareja sobre el uso del condón, las mismas variables resultaron ser predictoras, excepto la intención para usarlo en su próximo encuentro sexual.

En lo que respecta al componente de información y motivación, los datos concuerdan con lo reportado en diversas investigaciones. Si las personas cuentan con datos sobre cómo prevenir el VIH, será más probable que tengan los argumentos para solicitar el uso del condón (Ehon, 2008; Liu et al., 2014). Otro dato relevante en la presente investigación es que no se evalúa la influencia directa de las variables psicosociales (información y motivación) sobre el uso del condón en relaciones sexuales debido a que hay evidencia empírica que demuestra que tener creencias favorables hacia el uso del condón, niveles altos de conocimientos sobre el VIH/sida, percepción del riesgo e intención para usar el condón en el acto sexual no necesariamente implican que se use el condón en las relaciones sexuales (Lapeira, Acosta y Vásquez, 2016; Oliveira et al., 2012). Algunas explicaciones al respecto indican que usar el condón no solo depende de la percepción favorable, sino de las habilidades que se poseen para ello (Farmer y Meston, 2006; Miller 2006). De esta manera, en la presente investigación se buscó adicionalmente conocer cómo es que las variables de información y motivación pueden influir sobre las habilidades. De esta forma, se necesita, por un lado, evaluar en muestras similares si hay consistencia en las variables que predicen las habilidades $\mathrm{y}$, por otro lado, analizar si hay otros factores que pueden coadyuvar al desarrollo de las mismas, centrando el análisis en las habilidades que posee la persona para tener un comportamiento preventivo.

Otro aspecto importante de considerar es que la varianza explicada en cada uno de los modelos de regresión fue baja, si bien, de acuerdo con Kline (2011), obtener un valor alto permite predecir de una mejor manera los futuros resultados. Cuando se alcanzan valores estadísticamente significativos pero con valores bajos, es necesario incluir otras variables que pueden incrementar la varianza explicada. Con base en lo anterior, se sugiere evaluar en muestras similares si tales variables siguen prediciendo la frecuencia del uso del condón, e incorporar en la evaluación otras habilidades que se relacionan con ello, como por ejemplo tomar decisiones para tener una conducta preventiva (Vargas, Henao y González, 2007) o usar correcta y consistentemente el condón $(\mathrm{Ca}-$ milleri et al., 2015).

De igual forma, se requiere que para la evaluación de las habilidades no únicamente se consideren las medidas de autorreporte centradas en la percepción de qué y cómo lo hacen (Lameiras, Faílde, Bimbela y Alfaro, 2008; Sanders et al., 2012), sino más bien considerar la evaluación mediante la observación directa en situaciones simuladas sobre cómo lo hacen, ya que se ha visto que hay diferencias considerables entre lo que dicen y lo que realmente hacen cuando se evalúa su ejecución (Miller, 2006; Robles, Rodríguez, Frías y Moreno, 2014). Este punto es relevante dado que, en la medida que se tengan indicadores más fiables de su evaluación, se puede tener una mayor certeza de qué y cómo entrenar las citadas habilidades para lograr que las personas tengan sexo seguro y reduzcan así los índices de infección por VIH.

A modo de conclusión, tal y como lo mencionan Tulloch, McCaul, Milternberg y Smith (2004) y Pérez y Pick (2006), el entrenamiento en habilidades es fundamental para que las personas usen condón en sus relaciones sexuales. En el caso de las personas evaluadas en la presente investigación, es necesario el entrenamiento en habilidades para negociar el uso del condón, y asimismo en las de comunicación sobre cuestiones sexuales con la pareja. 


\section{REFERENCIAS}

Añaños, F. y Jiménez, F. (2016). Población y contextos sociales vulnerables: la prisión y el género al descubierto. Papeles de Población, 22(87), 1-39.

Asare, M. (2015). Using the Theory of Planned Behavior to determine the condom use behavior among college students. American Journal of Health Studies, 30(1), 43-50.

Austin, P. y Steyerberg, E. (2015). The number of subjects per variable required in linear regression analyses. Journal of Clinical Epidemiology, 68(6), 627-636.

Camilleri, M., Kohut, T. y Fisher, W. (2015). Condom use behavioural skills mediate the relationship between condom use motivation and condom use behaviour among young adult heterosexual males: An information-motivation-behavioural skills analysis. The Canadian Journal of Human Sexuality, 24(2), 131-140.

Centro Nacional para la Prevención y Control del VIH y el sida (CENSIDA) (2016). El condón (preservativo). México: CENSIDA. Recuperado de https://www.gob.mx/censida/articulos/el-condon-preservativo?idiom=es.

Centro Nacional para la Prevención y Control del VIH y SIDA (CENSIDA) (2018). Vigilancia epidemiológica de casos de VIH/SIDA en México, Registro Nacional de Casos de SIDA, Actualización al 2do. Trimestre del 2018. México: CENSIDA. Recuperado de https://www.gob.mx/cms/uploads/attachment/file/391168/RN_2do_trim_2018.pdf.

Comisión Nacional de los Derechos Humanos (CNDH) (2012). Los derechos humanos de las personas que viven con VIH o SIDA en reclusión ( $1^{\mathrm{a}}$ ed.). México: CNDH. Recuperado de http://www.cndh.org.mx/sites/all/doc/cartillas/17_Cartilla_DH_VIHSida_Reclusion.pdf.

Cutiño, S. (2015). Algunos datos sobre la realidad del tratamiento en las prisiones españolas. Revista Electrónica de Ciencia Penal y Criminología, 17(11), 1-41.

Eggers, A., Aarø, L., Bos, A., Mathews, C., Kaaya, S., Onya, H. y Vries, H. (2015). Sociocognitive predictors of condom use and intentions among adolescents in three sub-Saharan sites. Archives of Sexual Behavior, 45(2):353-365. doi: 10.1007/ s1058-015-0525-1.

Ehon, A. (2008). Assessing sexual communication and condom negotiation among university undergraduate students in sexual relationship; in the era of HIV/AIDS. Ife Psychologia, 16(2), 216-239.

Escaff, E., Estévez, M., Feliú, M. y Torrealba, C. (2013). Consecuencias psicosociales de la privación de la libertad en imputados inocentes. Revista de Criminalidad, 55(3), 291-308.

Farmer, M. y Meston, C. (2006). Predictors of condom use self-efficacy in an ethnically diverse university sample. Archives of Sexual Behavior, 35(3), 313-326.

Fishbein, M. (2000). El papel de la teoría en la prevención del VIH. AIDS Care, 12(3), 273-278. doi: 10.1080/09540120050042918.

Fisher, J. y Fisher, W. (1992). Changing aids-risk behavior. Psychological Bulletin, 3(3), 455-474.

Galindo, J. (2012). Vulnerabilidad en salud de los presos del Penal de Villahermosa, Cali, Colombia. Tesis inédita de licenciatura. Cali (Colombia): Universidad ICESI.

Icunacuri, M. (2010). Comunicación sexual en adolescentes y su implicación en la consistencia del uso del condón. Enseñanza e Investigación en Psicología, 15(1), 107-129.

Kerlinger, F. y Lee, H. (2002). Investigación del comportamiento. México: McGraw-Hill/ Interamericana.

Kiene, S.M., Fisher, W.A., Shuper, P.A., Cornman, D.H., Christie, S., MacDonald, S., Pillay, S., Mahlase, G. y Fisher, J.D. (2013). Understanding HIV transmission risk behavior among HIV-infected South Africans receiving antiretroviral therapy: An information-motivation-behavioral skills model analysis. Health Psychology, 32(8), 860-868. doi: 10.1037/a0030554.

Kline, R.B. (2011). Principles and practice of structural equation modeling. New York: The Guilford Press.

Lameiras, M., Faílde, J., Bimbela, J. y Alfaro, N. (2008). Uso del preservativo masculino en las relaciones con coito vaginal de jóvenes españoles entre catorce y veinticuatro años. Diversitas: Perspectivas en Psicología, 4(2), 401-415.

Lapeira, P., Acosta, D. y Vásquez, M.M. (2016). Conocimientos, creencias y prácticas de los adolescentes de la cultura caribe en anticoncepción. Revista CUIDARTE, 7(1), 1204-1209.

Leddy, A., Chakravarty, D., Dladla, S., de Bruyn, G. y Darbes, L. (2016). Autoeficacia de la comunicación sexual, normas masculinas hegemónicas y uso del condón entre parejas heterosexuales en Sudráfrica. Biblioteca Nacional de Medicina de EE.UU. 28(2), 228-233. doi: 10.1080/09540121.2015.1080792.

Liu, Z., Wei, P., Huang, M., Liu, Y., Li, L., Gong, X., Chen, J. y Li, X. (2014). Determinants of consistent condom use among college students in China: Application of the Information-Motivation-Behavior Skills (IMB) model. PLOS ONE, 9(9), 1-9. doi: 10.1371/journal.pone.0108976.

Marsh, H., Morin, A., Parker, P. y Kaur, G. (2014). Exploratory structural equation modeling: An integration of the best features of exploratory and confirmatory factor analysis. Annual Review of Clinical Psychology, 10(1), 85-110.

McGrane, H., Mittal, M., Elder, H. y Carey, M. (2016). Relationship factors and condom use among women with a history of intimate partner violence. AIDS Behavior, 20(1), 225-234. doi: 10.1007/s10461-015-1189-5. 
Méndez, I., Namihira, D., Moreno, L. y Sosa, C. (2001). El protocolo de investigación. México: Trillas.

Miller, K. (2006). Correct and consistent use of condoms in preventing STDs. American Family Physician, 73(4), 703-704.

Oliveira, A., Gomes, S., Amorim, P., Santos, A., Rodrigues, E. y Bezerra, A. (2012). Knowledge, attitude and practices regarding condom use among women prisoners: the prevention of STD/HIV in the prison setting. Revista da Escola de Enfermagem, 46(3), 707-714.

Organización Mundial de la Salud (2018). Temas de salud: salud sexual. Ginebra: OMS. Recuperado de http://www.who.int/ topics/sexual_health/es/.

Pérez, C. y Pick, S. (2006). Conducta sexual protegida en adolescentes mexicanos. Interamerican Journal of Psychology, 40(3), 333-340.

Pinos, G., Pinos, V., Palacios, M., López, S., Castillo, J., Ortiz, W., Jerves, E. y Enzlin, P. (2017). Conocimientos y actitudes hacia la sexualidad y educación sexual en docentes de colegios públicos. Actualidades Investigativas en Educación, 17(2), 1-22. doi: 10.15517/aie.v17i2.28671.

Programa Conjunto de las Naciones Unidas sobre el VIH/Sida (ONUSIDA) y Organización Mundial de la Salud (OMS) (2007). Informe Anual 2007. Ginebra: ONUSIDA/OMS.

Robles, S. y Díaz-Loving, R. (2011). Autoeficacia para usar condón ante situaciones de riesgo. En S. Robles y R. Díaz-Loving (Eds.): Validación de la Encuesta Estudiantil sobre Salud Sexual (EESS) (pp. 79-93). México: Universidad Nacional Autónoma de México.

Robles, S., Frías, B., Moreno, D., Rodríguez, M. y Barroso, R. (2011). Conocimientos sobre VIH/SIDA, comunicación sexual y negociación del uso del condón en adolescentes sexualmente activos. Revista Electrónica de Psicología Iztacala, 14(4), 317-340.

Robles, S., Rodríguez, M., Frías, B. y Moreno, D. (2014). Indicadores del uso eficaz del preservativo. Revista Puertorriqueña de Psicología, 25(2), 244-258.

Robles, S., Solano, R., Díaz, R., Moreno, D., Frías, B., Rodríguez, M. y Barroso, R. (2012). Efectos de un programa de prevención de problemas de salud sexual sobre variables psicosociales y conductuales en adolescentes sin experiencia sexual. Revista Electrónica de Psicología Iztacala, 15(3), 1129-1155.

Rubio, F. (2013). Aspectos psicológicos de las personas encarceladas y/o excarceladas. Nómadas, 37(1), 1-21.

Sánchez, R., Robles, S. y Enríquez, D. (2015). Factores asociados al uso del preservativo en adolescentes mexicanos en conflicto con la ley. Revista Digital Internacional de Psicología y Ciencia Social, 1(1), 114-125.

Sanders, S.A., Yarber, W.L., Kaufman, E.L., Crosby, R.A., Graham, C.A. y Milhausen, R.R. (2012). Condom use errors and problems: a global view. Sexual Health, 9(1), 81-95.

Tulloch, H., McCaul, K., Miltenberger, R. y Smyth, J. (2004). Partner communication skills and condom use among college couples. Journal of American College Health, 52(6), 263-267.

Uribe, J., Aguilar, J., Zacarías, X. y Aguilar, A. (2015). Modelos explicativos del uso del condón en las relaciones sexuales de adolescentes. Acta de Investigación Psicológica, 5(1), 1904-1915.

Uribe, J., Riaño, M., Bonilla, N., Carrillo, S., Hernández, Y. y Bahamon, M. (2017). Percepción de autoeficacia vs. rechazo del uso del condón en las prácticas sexuales de mujeres y hombres jóvenes. Psicoagente, 20(37), 25-35. doi: 10.17081/psico.20.37.2416.

Vargas, E., Henao, J. y González, C. (2007). Toma de decisiones sexuales y reproductivas en la adolescencia. Acta Colombiana de Psicología, 10(1), 49-63. 